OPEN ACCESS

Edited by:

Haruki Kitazawa

Tohoku University, Japan

Reviewed by:

Gabriela Del Valle Perdigon, CERELA-CONICET, Argentina

Jieliang Li,

Temple University, USA

*Correspondence:

Stine Indrelid

stine.indrelid@nmbu.no

${ }^{\dagger}$ Present Address:

Charlotte Kleiveland

Smerud Medical Research Norway AS, Oslo, Norway

Specialty section:

This article was submitted to Microbial Immunology,

a section of the journal

Frontiers in Microbiology

Received: 24 November 2016 Accepted: 15 February 2017 Published: 28 February 2017

Citation:

Indrelid S, Kleiveland $C$, Holst $R$ Jacobsen M and Lea T (2017) The

Soil Bacterium Methylococcus capsulatus Bath Interacts with Human Dendritic Cells to Modulate Immune Function. Front. Microbiol. 8:320. doi: 10.3389/fmicb.2017.00320

\section{The Soil Bacterium Methylococcus capsulatus Bath Interacts with Human Dendritic Cells to Modulate Immune Function}

\author{
Stine Indrelid ${ }^{1,2 *}$, Charlotte Kleiveland ${ }^{2 \dagger}$, René Holst ${ }^{1}$, Morten Jacobsen ${ }^{1,2}$ and Tor Lea ${ }^{2}$ \\ ${ }^{1}$ Research and Innovation, Østfold Hospital Trust, Kalnes, Norway, ${ }^{2}$ Department of Chemistry, Biotechnology and Food \\ Science, Norwegian University of Life Sciences, Aas, Norway
}

The prevalence of inflammatory bowel disease (IBD) has increased in Western countries during the course of the twentieth century, and is evolving to be a global disease. Recently we showed that a bacterial meal of a non-commensal, non-pathogenic methanotrophic soil bacterium, Methylococcus capsulatus Bath prevents experimentally induced colitis in a murine model of IBD. The mechanism behind the effect has this far not been identified. Here, for the first time we show that $M$. capsulatus, a soil bacterium adheres specifically to human dendritic cells, influencing DC maturation, cytokine production, and subsequent $T$ cell activation, proliferation and differentiation. We characterize the immune modulatory properties of $M$. capsulatus and compare its immunological properties to those of another Gram-negative gammaproteobacterium, the commensal Escherichia coli K12, and the immune modulatory Gram-positive probiotic bacterium, Lactobacillus rhamnosus GG in vitro. M. capsulatus induces intermediate phenotypic and functional DC maturation. In a mixed lymphocyte reaction $M$. capsulatus-primed monocyte-derived dendritic cells (MoDCs) enhance T cell expression of CD25, the $\gamma$-chain of the high affinity IL-2 receptor, supports cell proliferation, and induce a T cell cytokine profile different from both $E$. coli K12 and Lactobacillus rhamnosus GG. M. capsulatus Bath thus interacts specifically with MoDC, affecting MoDC maturation, cytokine profile, and subsequent MoDC directed $T$ cell polarization.

Keywords: dendritic cells (DC), old friends hypothesis, immune modulation, environmental bacteria, DC activation, $T$ cell polarization, immunobiotics, soil bacteria

\section{IMPORTANCE}

There has been a growing interest in probiotics for treating both IBD, allergies, and autoimmune diseases, and considerable effort has been invested in identifying novel probiotics aimed for treating immune pathologies. Typically, candidate probiotic bacteria has been of human or animal origin, and a host-associated lifestyle is assumed to be a prerequisite for developing immuneregulatory functions. Here we describe immune modulatory functions of a non-commensal soil bacterium previously shown to exhibit anti-inflammatory effects in a murine colitis model pointing to environmental bacteria as a new and untapped source of bacteria for modulating immune responsiveness. 


\section{INTRODUCTION}

Although microbes are associated with all epithelial surfaces of animal hosts, the highest number, and most diverse microbial populations are found in the intestines. Some 10-100 trillion microbes colonizes the human gastrointestinal tract, with the highest numbers present in the colon (Turnbaugh et al., 2007). The physiology of these microbes and their hosts is closely connected and mutually regulated (Brown et al., 2013). The host shapes the composition of the intestinal microbiota at species and community levels by supplying nutrients and by producing antimicrobial peptides. The human microbiota in return, adds to the metabolic, and biochemical activities of the host and play essential roles in the development and differentiation of the host intestinal epithelium, the immune system, and in the maintenance of mucosal homeostasis (Nicholson et al., 2012; Sommer and Backhed, 2013).

Only a single layer of epithelial cells separates the luminal contents and microbial community from underlying tissues, and the epithelial barrier therefore provides a possible entry point for opportunistic pathogens into the body. The host must maintain a mutualistic relationship with the commensal microbiome, while retaining protective responsiveness against pathogenic bacteria. To achieve this it must preserve epithelial integrity and regulate pro- and anti-inflammatory signaling, in an appropriate manner. Homeostasis is maintained through continuous and dynamic interactions and communication between the intestinal microbiota, the epithelium, and immune cells in the intestinal mucosa.

The regulatory interactions that exist between multicellular organisms and the microbial world are not necessarily limited to those between commensals and their hosts. The increasing prevalence of inflammatory bowel disease and autoimmune diseases in the western world has been associated with reduced exposure to helminths and environmental microorganisms from soil, water, and fermenting vegetables (Rook, 2007). The "hygiene hypothesis" was forwarded as a result of studies coherent with the idea that postnatal infections may be protective against allergy later in life, and that such protection may be lost in the presence of modern hygiene (Strachan, 1989, 2000). The related "old-friend hypothesis" explains the striking increase in chronic inflammatory disorders as largely being due to reduced contact with microorganisms that we have coevolved with, and therefore depend on, for proper immune development and regulation (Rook, 2010). In this context both pathogenic bacteria, the commensal microbiota, pseudo-commensals, and even the environmental microbiota may be essential regulatory components of the mammalian immune system. An increased mechanistic understanding of how such microbes and microbial products affect immune homeostasis may form a basis for developing novel tools for modulating immune responses in chronic inflammatory disorders.

Recently we demonstrated that a bacterial meal of the Gram-negative soil bacterium, Methylococcus capsulatus Bath, ameliorates dextran sulfate sodium (DSS) induced colitis in mice (Kleiveland et al., 2013). The study points to a potential for non-commensal environmental bacteria in protecting against experimental colitis in mammals, but the mechanisms behind these effects have not been identified. Both live and heatkilled probiotic bacteria have previously been shown to protect against experimental colitis (Mileti et al., 2009; Sang et al., 2014; Toumi et al., 2014; Souza et al., 2016). Proposed modes of action include competitive pathogen exclusion, production of antimicrobial substances, gut flora modulation, modulatory effects on epithelial barrier integrity, regulatory effects on innate, and adaptive immunity and effects on epithelial development and survival (Bermudez-Brito et al., 2012). However, direct effects on dendritic cells (DCs) with subsequent effects on cytokine production and $\mathrm{T}$ cell development is expected to be a common mode of action for those probiotic strains able to modulate adaptive immunity (Bienenstock et al., 2013).

DCs are professional antigen presenting cells that play a key role in both innate and adaptive immune responses (Steinman, 2012). Intestinal DCs expresses pattern recognizing receptors (PRRs) to recognize various microbial structures and can distinguish between microbe-associated molecular patterns (MAMPs) of even closely related organisms to initiate specific and appropriate response. The capacity of DCs to activate naïve $\mathrm{T}$ cells inducing $\mathrm{T}$ cell expansion and polarization, position DCs as critical mediators of host immune tolerance, and inflammatory responses (Mann et al., 2013).

The dietary inclusion of $M$ capsulatus Bath in DSS-colitis model affected the intestinal epithelium through increased cell proliferation and mucin production, suggesting beneficial effects on gut barrier function. However, direct effects on cells of the immune system was not evaluated in that study. Here, for the first time, we show that $M$. capsulatus Bath, a noncommensal environmental bacterium, specifically and strongly adheres to murine and human DCs, an immune cell type central in regulating both innate and adaptive immunity. We compare the immune modulatory effects of $M$. capsulatus Bath to those of the Gram-negative commensal Escherichia coli K12, a non-pathogenic E. coli strain originally isolated from stool of a diphtheria patient (Agency USEP, 1997), and the well characterized Gram-positive probiotic bacterium Lactobacillus rhamnosus GG. The interaction between DC and M. capsulatus leads to functional activation of the DCs, affects DC cytokine profile, improves $\mathrm{T}$ cell activation, and proliferation and drive $\mathrm{T}$ effector cell polarization in vitro.

\section{MATERIALS AND METHODS}

\section{Bacterial Strains and Culture Conditions}

M. capsulatus strain (Bath) (NCIMB 11132, Aberdeen, UK) were grown in nitrate mineral salts medium (Whittenbury et al., 1970) with a head-space of $75 \%$ air, $23.75 \% \mathrm{CH}_{4}$, and $1.25 \% \mathrm{CO}_{2}$ at $45^{\circ} \mathrm{C}$ and $200 \mathrm{rpm}$. E. coli strain $\mathrm{K} 12$ was kindly provided by Department of Bacteriology, the Norwegian Veterinary Institute, Norway. E. coli K12 (Blattner et al., 1997) was grown in LB medium (Oxoid Ltd., Basingstoke, United Kingdom) at $37^{\circ} \mathrm{C}$ and 200 rpm. L. rhamnosus GG was grown in MRS medium (Oxoid Ltd.) anaerobically at $37^{\circ} \mathrm{C}$ without shaking. 


\section{Cells and Culture Conditions}

Human erythrocyte- and plasma depleted blood were obtained from healthy volunteers from Ostfold Hospital Trust, Fredrikstad, Norway in accordance with institutional ethical guidelines and with approval from the Regional Committee of Medical and Health Research Ethics with written informed consent from all subjects. All subjects gave written informed consent in accordance with the Declaration of Helsinki. Peripheral blood mononuclear cells (PBMCs) were isolated by density gradient centrifugation on a Lymphoprep gradient (Fresenius Kabi). Human T cells were isolated from PBMCs by negative selection using Dynabeads Untouched Human $\mathrm{T}$ Cells Kit (Thermo Fisher). CD14 ${ }^{+}$cells were isolated by positive selection using human CD14 MicroBeads (Miltenyi Biotech). To develop immature monocyte-derived dendritic cells (MoDCs) $\mathrm{CD} 14^{+}$cells were cultivated for 6 days in RPMI 1640 medium supplemented with 10\% heat inactivated fetal calf serum and $25 \mu \mathrm{g} / \mathrm{ml}$ gentamicin sulfate (Lonza), $1 \mathrm{mM}$ sodium pyruvate and $100 \mu \mathrm{M}$ non-essential amino acids (both from PAA Laboratories), $25 \mathrm{ng} / \mathrm{ml}$ interleukin 4 and $50 \mathrm{ng} / \mathrm{ml}$ granulocyte macrophage colony stimulating factor (both from ImmunoTools).

\section{Bacterial Stimulation, Cytokine Analysis, and Immune Phenotyping of MoDCs}

For immune phenotyping and DC cytokine analysis MoDCs were primed for $24 \mathrm{~h}$ by bacteria in a ratio of $1: 10$ (MoDC: bacteria) or by a maturation cocktail of $15 \mathrm{ng} / \mathrm{ml}$ TNF- $\alpha$ (ImmunoTools), $100 \mathrm{ng} / \mathrm{ml} \mathrm{LPS} \mathrm{and} 5 \mu \mathrm{g} / \mathrm{ml} \mathrm{PGE2} \mathrm{(Sigma-}$ Aldrich). Culture supernatants were harvested and stored at $-20^{\circ} \mathrm{C}$ then analyzed for cytokines by ProcartaPlex Multiplex immunoassay (eBioscience). TGF- $\beta$ and IL- 6 was measured by ELISA kits (eBiosciences and PeproTech respectively). MoDCs were also harvested and stained using PE-conjugated mouse antihuman CD80 antibodies, PE-Cy5 conjugated mouse anti-human CD83, and PE-Cy5 conjugated mouse anti-human CD40 (all from BD Biosciences). For viability testing cells were stained by $1 \mu \mathrm{g} / \mathrm{ml} \mathrm{PI}$ and analyzed by flow cytometry.

\section{DC-T Cell Co-cultures for Cytokine Analysis and Immunophenotyping}

To induce antigen specific $\mathrm{T}$ cell responses a modified mixed leukocyte culture system (MLC) were used with MoDC as stimulator cells and purified peripheral blood $\mathrm{T}$ cells as responder cells. MoDCs, either unprimed or primed by UV-inactivated bacteria in a ratio of 1:100 (MoDC:bacteria) for $24 \mathrm{~h}$, were coincubated with allogeneic T cells from two different donors (1:10 ratio between MoDCs and T cells). For cytokine analysis cells were grown in 48 well plates. After 5 days culture supernatants were harvested and $\mathrm{T}$ cells phenotyped by flow cytometry using FITC-conjugated anti-human CD4 and APC-conjugated anti-CD25 (both from Miltenyi Biotech). Fluorescence was detected by a MACSQuant flowcytometer and analyzed using the MACSQuantify software (both from Miltenyi Biotech). Cytokine concentrations in culture supernatant were measured by ProcartaPlex Multiplex immunoassay (eBioscience).

\section{T Cell Proliferation Assay}

MoDCs were primed for $24 \mathrm{~h}$ with UV-inactivated $M$. capsulatus 1:100 (DC:bacteria) in Nunc ${ }^{\mathrm{TM}} \mathrm{UpCell}^{\mathrm{TM}}$ plates (Thermo Fisher). After 24 of stimulation the MoDCs were harvested, washed and co-incubated with allogenic human T cells in 96-well plates in a ratio of 1:10 (DC:T cells). Next day recombinant human IL2 was added to each well to a final concentration of $10 \mathrm{U} / \mathrm{ml}$. After $96 \mathrm{~h}$ of co-culture cells were pulsed by $\left[{ }^{3} \mathrm{H}\right]$-thymidine $(1$ $\mu \mathrm{Ci}$, Perkin Elmer) for $18.5 \mathrm{~h}$. Cells were harvested onto glassfiber filters and incorporated thymidine determined by liquid scintillation counting using a TopCount $\mathrm{NXT}^{\mathrm{TM}}$ Luminometer (Packard BioScience Company).

\section{Scanning Electron Microscopy (SEM)}

Immature MoDCs were co-cultivated with $M$. capsulatus Bath in 1:100 ratio (cells:bacteria) in medium free of antibiotics for $2-4 \mathrm{~h}$ in a humified atmosphere with $5 \% \mathrm{CO}_{2}$. Cells were washed twice by phosphate buffered saline (PAA Laboratories), fixed with $4 \%$ PFA and 2.5\% glutaraldehyde (1:1) for $20 \mathrm{~min}$ at room temperature. Cells were washed again, dehydrated in a graded ethanol series and dried using a critical point dehydrator (CPD030 BalTec). Samples were coated with $\sim 500 \AA \mathrm{Pt}$ in a sputter coater (Polaron SC7640, Quorum technologies) and analyzed using an EVO-50 Zeiss microscope (Carl Zeiss AG).

\section{Confocal Imaging}

Immature MoDCs were generated from $\mathrm{CD} 14^{+}$monocytes as described above. $1 \times 10^{8} / \mathrm{ml}$ M. capsulatus Bath were stained by $10 \mu \mathrm{M}$ CFSE in PBS. CFSE-stained bacteria were coincubated with immature MoDCs in a ratio of 1: 100 cellsbacteria. Cells were washed, fixated in PBS with $1 \%$ formalin then washed twice before coverslip was mounted on object slide with ProLong Diamond Antifade Mountant with DAPI (Thermo Fisher Scientific). Samples were scanned under a Zeiss LSM510 META confocal microscope (Carl Zeiss). Confocal stacks were acquired with $\mathrm{z}$-spacing of $0.2 \mu \mathrm{m}$.

\section{Statistical Analysis}

Data were sampled in hierarchical structure, with multiple measurements per individual. This violates the assumption of independent measurements underlying ANOVA and conventional linear regression. This issue was remedied by analyzing the data using a mixed effects linear model, in which each individual acted as a random effect. Box-Cox analyses were used for finding suitable normalizing transformations. Data were analyzed on the log-scale and subsequently back-transformed for interpretation. All analyses were controlled by residual plots and Shapiro-Wilks test for normality.

\section{RESULTS}

\section{M. capsulatus Bath Adheres Specifically to MoDC}

A bacterial protein preparation of $M$. capsulatus Bath was previously found to have anti-inflammatory effects in a murine model of colitis (Kleiveland et al., 2013). When studying possible immune modulatory effects on immune cells, we 
observed that bacteria clustered around a small subset of cells in peripheral blood mononuclear cell preparations (Figure 1A). The appearance and low frequency of the target cells were consistent with the size and expected frequency of DCs among PBMCs. To determine whether the target cells were in fact DCs we incubated $M$. capsulatus Bath with $\mathrm{CD} 14^{+}$monocytes or MoDCs generated from $\mathrm{CD} 14^{+}$monocytes in the presence of IL-4 and GM-CSF. M. capsulatus did not bind to CD14 ${ }^{+}$ monocytes (Figure 1B), but quickly associated with dendritic cells (Figure 1C). The interaction between M. capsulatus Bath and MoDCs was further visualized by scanning electron microscopy (SEM) showing M. capsulatus Bath in large clusters on MoDCs after $3 \mathrm{~h}$ of co-incubation, even after several washes with PBS (Figure 1D).

To study binding kinetics we co-incubated CFSE-stained bacteria with MoDCs. Cells were counterstained with DAPI and confocal microscopy was used to visualize interactions over time (Figure 2). M. capsulatus Bath were found in scattered association with cells after just $30 \mathrm{~min}$ of co-incubation, and after $2 \mathrm{~h}$ a large number of bacteria associated with most cells. Strikingly, after around $3 \mathrm{~h}$ of co-incubation M. capsulatus were typically found to cluster around the nucleus of the MoDCs. A large number of bacteria could be seen associated with cells up to $20 \mathrm{~h}$ after co-incubation. At $48 \mathrm{~h}$ bacteria were cleared from most cells although a few intact bacteria was found associated with cells up to $72 \mathrm{~h}$ after co-incubation (Figure 2).

\section{M. capsulatus Bath Induces Phenotypic and Functional Maturation of MoDCs}

Upon microbial stimulation, DCs undergo a program of maturation that endows them with capacity to activate naïve $\mathrm{T}$ cells, induce $\mathrm{T}$ cell expansion, and to polarize $\mathrm{T}$ cells toward effector subpopulations appropriate to the stimulus encountered. Mature DCs are characterized by expression of co-stimulatory molecules required for efficient $\mathrm{T}$ cell activation. We compared the ability of $M$. capsulatus Bath, Gram-positive, and Gramnegative control bacteria to induce MoDC activation as assessed by expression of costimulatory molecules like CD40, CD80, and CD83. MoDCs, either left unprimed or co-incubated with bacteria ( $M$. capsulatus Bath, L. rhamnosus GG, or E. coli K12) were stained for co-stimulatory molecules and maturation markers and analyzed by flow cytometry (Figure 3). Cells activated by a maturation cocktail containing TNF- $\alpha$, LPS, and $\mathrm{PGE}_{2}$ were used as a positive control. The maturation cocktail, $E$. coli K12, and M. capsulatus Bath induced upregulation of CD40, CD83, and CD80 in immature MoDCs. E. coli K12 was found to represent the most potent bacterial stimulus for inducing a mature DC phenotype compared to unprimed control cells, and induced expression of all activation markers. M. capsulatus Bath showed intermediate ability to induce MoDC maturation and elicited CD40 and CD80 expressions comparable to positive control, but a lower expression of CD83 compared to E. coli and the maturation cocktail (Figure 3). L. rhamnosus GG was a weak inducer of MoDC maturation, and produced a phenotype similar to unprimed cells. Cell viability, determined by PI staining, was similar between treatments suggesting that none of the strains asserted toxic effects on MoDCs (Data not shown).

\section{MoDCs Respond to Bacterial Stimulation by Eliciting Distinct Cytokine Profiles}

Depending on the nature of the stimuli, functionally mature DCs release cytokines promoting differentiation of naïve $\mathrm{T}$ cells into specific effector cell subsets. Since M. capsulatus and E. coli induced phenotypic maturation of MoDCs we wanted to see whether the bacteria also resulted in functional maturation characterized by cytokine release. Multiplex immunoassay and enzyme-linked immunosorbent assay (ELISA) was used to measure select cytokines in culture supernatants of MoDCs cocultivated with bacteria for $24 \mathrm{~h}$ (Figure 4). In general, and in accordance with the observed phenotypic activation of MoDC, E. coli $\mathrm{K} 12$ was the most potent inducer of cytokine production, and resulted in increased release of IL-1 $\beta$, IL-12p70, IL-10, TNF- $\alpha$, IL-2, IL-23, IFN- $\gamma$, and IL-6 compared to unprimed control. L. rhamnosus GG in comparison was the least potent inducer of cytokine production in MoDCs of the three tested bacteria (Figure 4). Incubation with M. capsulatus Bath resulted in intermediate levels of cytokines. Similar to $E$. coli-primed MoDCs, incubation with $M$. capsulatus enhanced the production of IL-12p70, IL-10, TNF- $\alpha$, IL-2, and IL-23 compared to unprimed MoDCs. However, M. capsulatus treatment in general resulted in lower cytokine levels than E. coli K12. M. capsulatusprimed cells produced substantially less IL-1 $\beta$, IL-6, IL-10, IL12p70, IL-23, and TNF- $\alpha$ than E. coli-primed cells, but the two bacteria induced similar levels of IL-2 from the MoDCs. TGF$\beta$ could not be detected in any of the co-cultures (data not shown). Thus, the interaction between M. capsulatus Bath and MoDCs resulted in both quantitative and qualitative differences in cytokine production compared to E. coli K12.

\section{M. capsulatus Bath Increases DC-Induced T Cell Activation and Proliferation}

Antigen recognition and a co-stimulatory signal through CD28 on $\mathrm{T}$ cells is required to induce functional $\mathrm{T}$ cell activation and clonal expansion. As the bacteria differently induced expression of DC co-stimulatory molecules, we examined the ability of bacteria-primed MoDCs to activate and induce proliferation in peripheral blood $\mathrm{T}$ cells. We co-incubated bacteria-primed MoDCs with allogenic T cells and measured expression of CD25 by flow cytometry. T cells co-cultivated with $M$. capsulatusprimed MoDCs expressed increased levels of CD25 compared to $\mathrm{T}$ cells cultivated with unprimed MoDCs or MoDCs primed by any of the control bacteria (Figure 5A). To test the ability of bacteria-treated MoDCs to induce proliferation in allogeneic T cells we measured DNA synthesis by $\left[{ }^{3} \mathrm{H}\right]$ thymidine incorporation. MoDCs activated by M. capsulatus were stronger supporters of $\mathrm{T}$ cell proliferation than MoDCs primed by any of the control bacteria (Figure 5B).

\section{MoDCs Primed by Different Bacteria Have Different Ability to Drive T Cell Differentiation}

Cytokines produced by mature DCs contribute to drive $\mathrm{T}$ cell differentiation into specific effector cell subsets. In order to evaluate functional effects of bacteria-treated MoDCs on $\mathrm{T}$ cell polarization, activated MoDCs were co-incubated with 

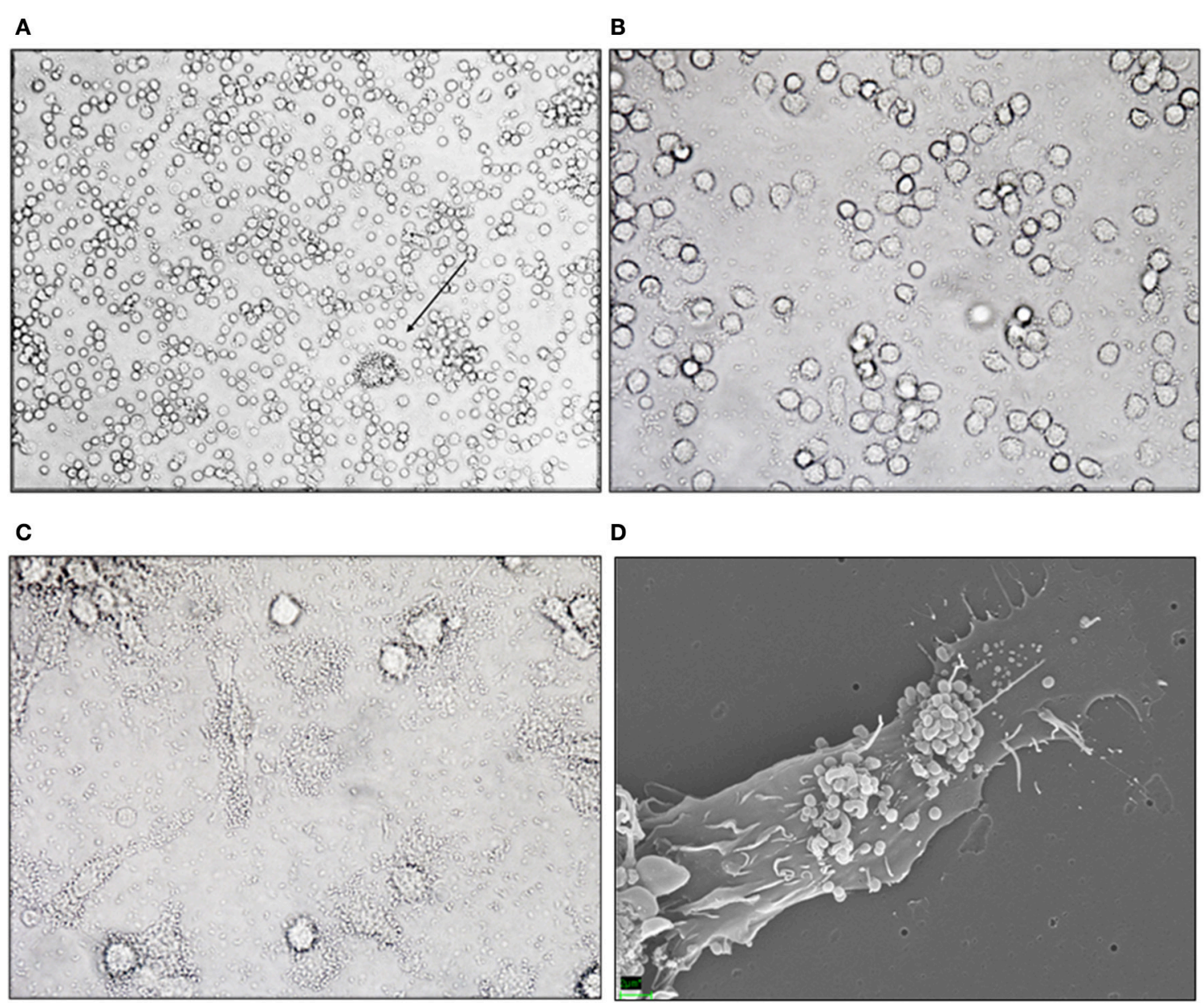

D

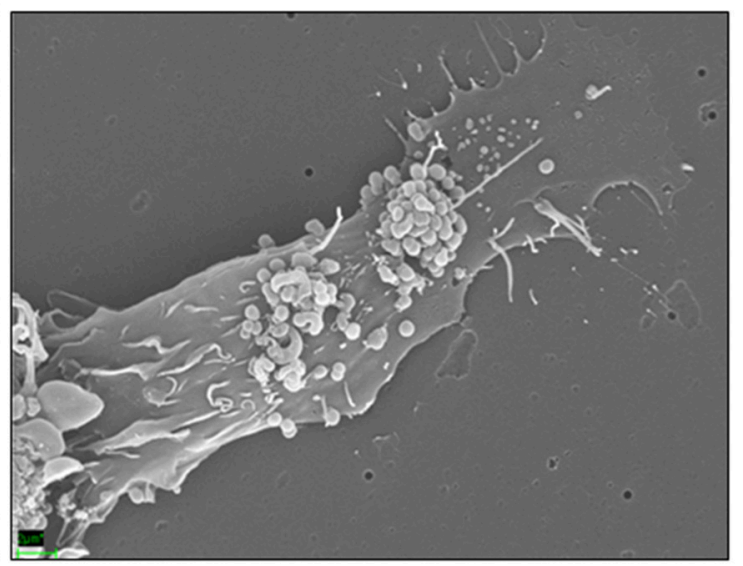

FIGURE 1 | M. capsulatus Bath interacts specifically with human MoDCs. (A-C) Light microscopy image of M. capsulatus Bath co-incubated (1:100 cells:bacteria) with human PBMC, CD14 ${ }^{+}$monocytes, or monocyte-derived dendritic cells without washing. M. capsulatus Bath clusters around low frequency-cells in PBMC (C) (arrow), but not CD14+ monocytes (B). In co-culture with MoDCs bacteria cluster around a majority of cells (C). (D) SEM electrograph showing M. capsulatus Bath adhering to human MoDCs after $3 \mathrm{~h}$ co-incubation.

allogeneic T cells. Culture medium was collected and analyzed for cytokines associated with different effector $\mathrm{T}$ cell subsets (Figure 6). MoDCs primed by any of the bacteria resulted in markedly reduced levels of typical Th2 cytokines like IL- 5 and IL-13. All bacteria further resulted in increased release of the Th1 cytokine IFN-gamma and IL-10, an anti-inflammatory cytokine produced by several effector $\mathrm{T}$ cell lineages, compared to the basal level produced by $\mathrm{T}$ cells co-incubated with unprimed MoDCs.

Although all bacteria shifted T cells toward a Th1 rather that a Th2 phenotype, a major difference was found between Gramnegative $M$. capsulatus Bath and E. coli $\mathrm{K} 12$ on the one hand and Gram-positive L. rhamnosus GG on the other. Compared to T cells co-cultivated with unprimed MoDCs only L. rhamnosustreated MoDCs resulted in significantly reduced release of IL-18, a proinflammatory cytokine that enhances innate immunity as well as Th1- and Th2-driven immune responses depending on cytokine milieu.
Conversely, only the Gram-negative bacteria $M$. capsulatus Bath and E. coli K12 gave significantly higher levels of the proinflammatory cytokines IL-6, TNF- $\alpha$, IL- $1 \beta$, and IL- $1 \alpha$. Both bacteria also increased IL-23, a cytokine linked to the generation and maintenance of Th17 cells, Th17 cytokines (IL-17A, IL-21, IL-22), Th22 cytokines (IL-22, TNF- $\alpha$ ), and Th9 cytokines (IL-9 and IL-21).

Not all differences could be attributed to dissimilarities between Gram-positive vs. Gram-negative bacteria, however. No significant difference was found between $E$. coli and $M$. capsulatus in their ability to induce Th1, Th22, or Th9 cells, as evaluated by IFN- $\gamma$, TNF- $\alpha$, IL-9, and IL-21, but compared to E. coli, M. capsulatus resulted in significantly less IL-23, Th17- associated cytokines IL-17A, and IL-22 as well as proinflammatory cytokines IL1- $\alpha$, IL- $1 \beta$, and IL- 6 and the antiinflammatory cytokine IL-10. Furthermore, reduction in Th2 cytokines IL-5 and IL-13 was lowest for M. capsulatus Bath primed co-cultures and E. coli and L. rhamnosus, but not $M$. 


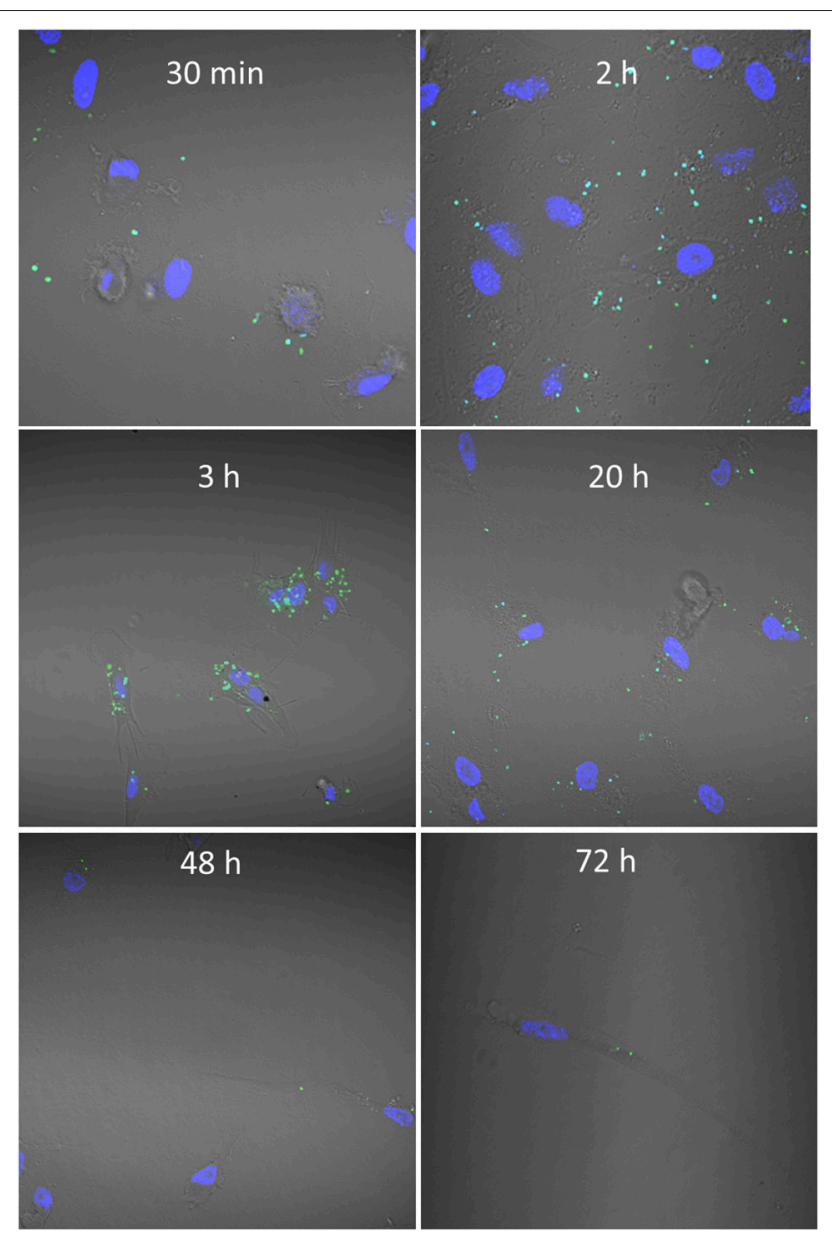

FIGURE 2 | Methylococcus capsulatus-DC interaction kinetics. Figure shows CFSE labeled $M$. capsulatus Bath (green) co-incubated with human MoDCs for $30 \mathrm{~min}$ to $72 \mathrm{~h}$. MoDC nuclei were counterstained with DAPI (blue) to aid visualization and interactions were visualized by confocal microscopy.

capsulatus Bath, reduced IL-1RA and lymphotoxin- $\alpha$ levels in the cultures. $M$. capsulatus thus induces a $\mathrm{T}$ cells response functionally distinct from both E. coli K12 and L. rhamnosus GG.

\section{DISCUSSION}

Previous studies have described protective properties of probiotic bacteria, commensals, and their metabolites against experimental colitis in animal models (Pils et al., 2011; Qiu et al., 2013; Toumi et al., 2014; Souza et al., 2016). Although a connection between chronic intestinal inflammation and a reduced exposure to bacteria from soils and water has been made (Rook, 2007), few studies have focused on immune modulatory effects of noncommensal environmental bacteria. Here we show that a soil bacterium previously shown to reduce symptoms of chemically induced colitis in mice (Kleiveland et al., 2013) specifically targets human dendritic cells in vitro, affecting DC maturation, $\mathrm{T}$ cell activation, proliferation, and differentiation. M. capsulatus Bath was found to adhere specifically to human DCs. To our knowledge, this is the first report of an environmental bacterium to target mammalian DCs to modulate immune function.

The realization that a soil bacterium interacts specifically with human DCs raises some important questions. The significance of the commensal microbiome in health and disease is increasingly recognized, and there is a growing interest in probiotics within the scientific and public community. However, the role of environmental bacteria in immune regulation has been underappreciated for understandable reasons. It is not difficult to imagine that commensals living in close connection with humans are also closely connected to human physiology (Sommer and Backhed, 2013). Similarly, there is a long history of probiotics in fermented food associated with longevity and health. In a modern world of reduced microbial diversity it may be less intuitive to connect environmental bacteria to regulation of human health. However, as emphasized by the "old friends" hypothesis, mammals are evolutionary linked not only to commensals and probiotics, but also to ambient microbes in both soil and water (Rook, 2010).

Not only have mammals evolved from environmental bacteria, but the mammalian immune system has developed in the presence of such microbes. Throughout evolution some of these microbes may have taken on functions for us, some may relay signals necessary for immune development, while others, because of our long evolutionary association, are recognized by the immune system as harmless and have taken on regulatory roles (Rook et al., 2004). For example, chronic exposure to environmental LPS and other bacterial components present in farm dust may protect against asthmatic disease (Schuijs et al., 2015) possibly by reducing the overall reactivity of the immune system.

M. capsulatus Bath is an environmental bacterium that has been isolated from soil, water, sewage, mud, and lake sediments. It does not require a host to survive and may therefore face no obvious selection pressure to express immune modulatory molecules. Nevertheless, as discussed by Casadevall and Pirofski (2007), soil is an extreme environment with rapidly changing conditions, and bacteria living in soils will encounter an enormous number of predators of different types: unicellular amoebas, slime molds, protists, nematodes, snails as well as larger animals. As they are likely to meet ever-changing conditions as well as predatory hosts with different types of receptors and antimicrobial defenses, soil dwellers have to carry a diverse array of characteristics including host cell adhesins and immune modulatory molecules as defense mechanisms against predators. It was beyond the scope of our study to identify the bacterial factors involved in adhesion. However, a computational and experimental analysis of the $M$. capsulatus secretome has previously identified $M$. capsulatus Bath protein homologs of adhesion proteins that are involved in microbe adhesion to host cells in other bacterial species (Indrelid et al., 2014), showing that candidate host interaction proteins are present in $M$. capsulatus Bath and may facilitate adhesion to DC.

The maturation state and cytokine profile of DCs is functionally important. Although DCs represent a heterogeneous group of antigen-presenting cells, they have traditionally been divided into immature and mature differentiation stages 


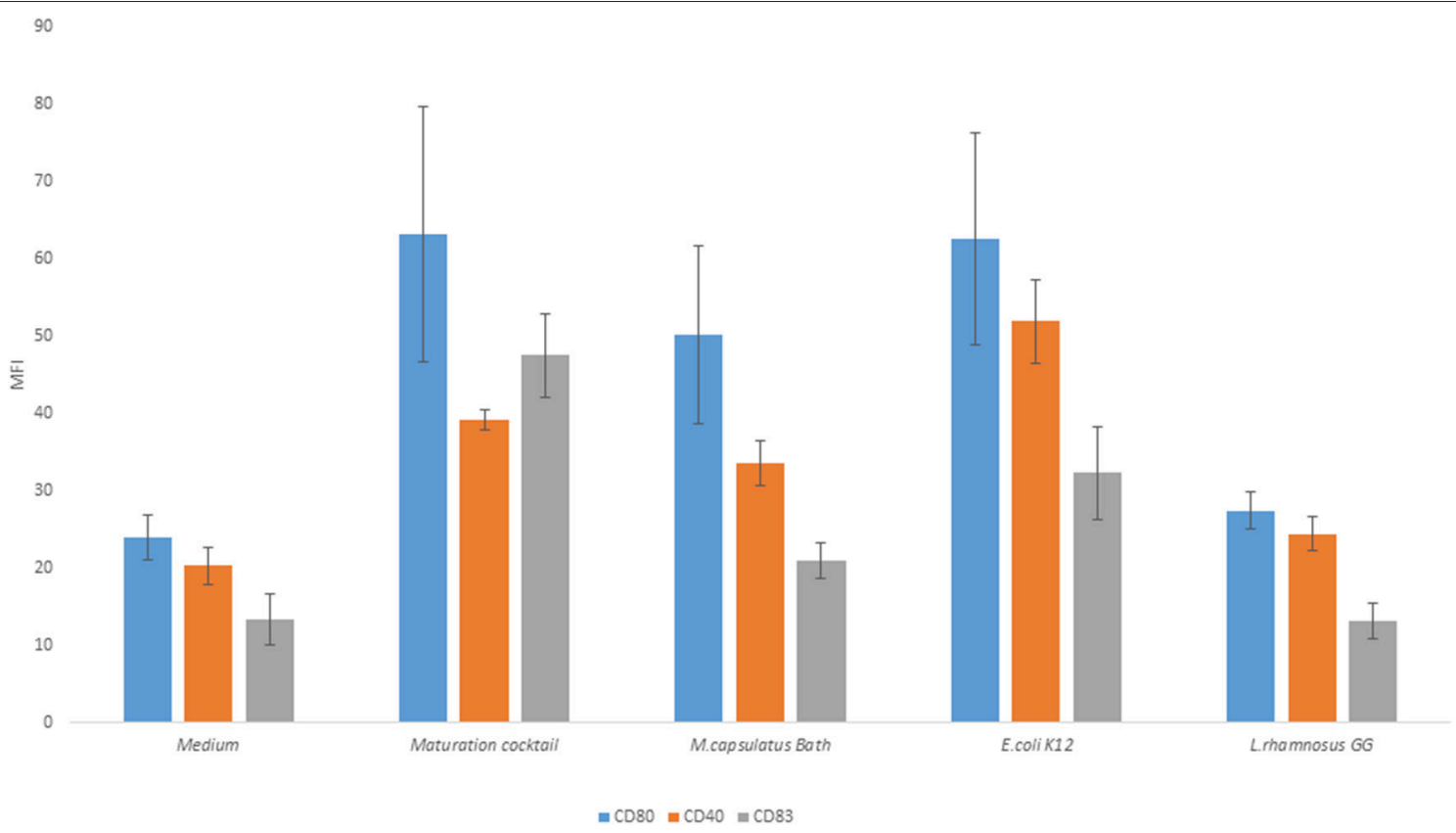

FIGURE 3 | M. capsulatus Bath and E. coli K12 induce maturation of MoDCs. Human MoDCs were either activated by a maturation cocktail of TNF- $\alpha$, PGE2, and LPS or co-incubated with bacteria (M. capsulatus Bath, E. coli K12, or L. rhamnosus GG) for 24 h. Cells were stained for CD80, CD83, and CD40 and analyzed by flow cytometry in this figure. Median fluorescence intensity (MFI) is reported. Error bars indicate standard error on median fluorescence intensity values from 6 different donors.

(Reis e Sousa, 2006). Immature DCs are characterized by low surface expression of major histocompatibility complex (MHC) class II molecules and co-stimulatory molecules (e.g., CD80, CD86, and CD40). However, when DCs encounter microbes, pattern-recognition receptors (PRRs) are triggered by microbe-associated molecular patterns resulting in major changes in gene expression and acquisition of a number of functional properties: antigen processing and presentation, migration, and T-cell co-stimulation (Dalod et al., 2014).

It has been proposed that pathogen, probiotic, and commensal bacteria can be divided into three distinct classes based on the extent of host response by DCs and other PRR expressing cells. MAMPs of pathogenic microorganisms tend to induce a strong host response, probiotics induce an intermediate response whereas commensal bacteria exhibit homeostatic control of the response (Lebeer et al., 2010). In the present study the non-commensal, non-pathogenic $M$. capsulatus Bath induced a DC response intermediate between the Gram-positive probiotic Lactobacillus rhamnosus GG and the commensal Gram-negative E. coli K12. Substantial differences were found between $M$. capsulatus Bath, L. rhamnosus GG and the E. coli $\mathrm{K} 12$ in their ability to induce phenotypical and functional maturation of monocyte-derived DCs. Toll like receptor 4 is expressed on MoDCs and recognize lipopolysaccharide (LPS), the major component of the outer membrane of Gram-negative bacteria (Schreibelt et al., 2010). LPS represents a strong stimulatory signal for inducing expression of co-stimulatory molecules and cytokine production in DCs (Verhasselt et al., 1997). Concordantly, E. coli K12 and M. capsulatus Bath were found to be stronger inducers of DC maturation and cytokine release compared to the Gram-positive L. rhamnosus. It has been suggested that probiotic bacteria modulate immune response by controlling the maturation of DCs and thereby the release of proinflammatory cytokines (Foligne et al., 2007). Both the Gramnegative bacteria tested in our study induced phenotypical and functional maturation. However, M. capsulatus Bath produced a less mature phenotype and substantially lower cytokine levels than E. coli K12. The fact that the Gram-negative $M$. capsulatus Bath results in a less mature phenotype and low levels of proinflammatory cytokines, suggests that similarly to probiotic bacteria $M$. capsulatus may modulate immunity through directing the maturation of DCs.

Interestingly, although priming with $M$. capsulatus resulted in a less mature MoDC phenotype than E. coli $\mathrm{K} 12$, it was found more efficient than both E. coli $\mathrm{K} 12$ and L. rhamnosus GG bacteria in inducing $\mathrm{T}$ cell activation and proliferation in the presence of interleukin 2, a growth factor necessary for cell cycle progression and clonal expansion (Smith, 1988). M. capsulatusprimed MoDCs enhanced T cell expression of CD25, the $\alpha$-chain of the trimeric high affinity IL-2 receptor explaining the increased proliferative $\mathrm{T}$ cell response compared to the other bacteria.

Whereas, co-stimulatory molecules on DCs and T cells are necessary for $\mathrm{T}$ cell activation and proliferation, $\mathrm{DC}$ cytokines are central in polarizing effector $\mathrm{T}$ cell development. In addition to antigen presentation and signaling through co-stimulatory molecules, cytokines provide a third signal for activation and differentiation of naïve $\mathrm{T}$ cells to effector cells. The nature of signal 3 depends on the triggering of particular PRRs by MAMPs 


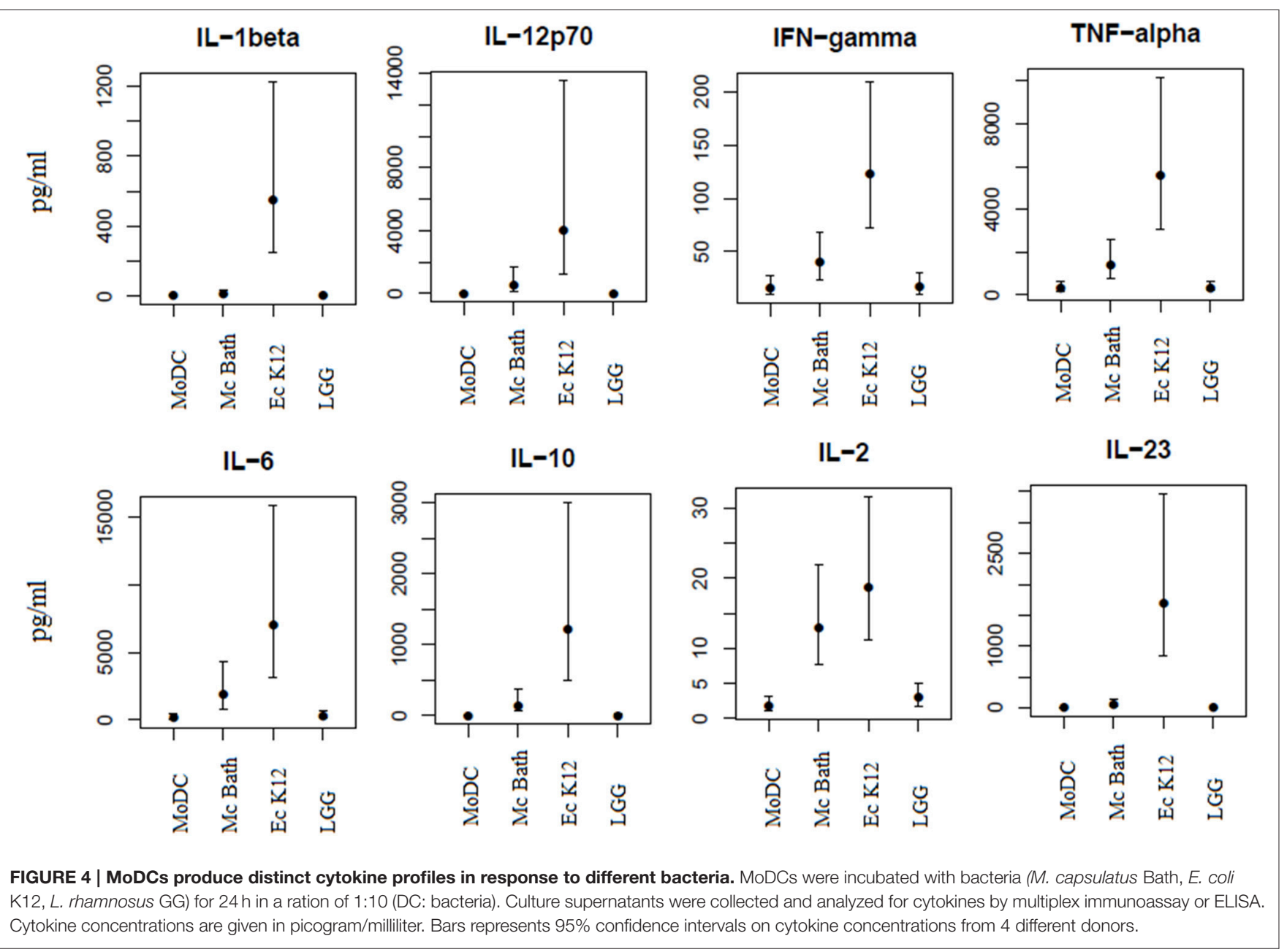

specific to the microbe encountered (Kapsenberg, 2003). Several DC-derived cytokines are important for $\mathrm{T}$ cell polarization into specific $\mathrm{T}$ cell subsets, e.g., IFN $\gamma$ and IL-12p70 are known to be important for Th1 polarization, IL-4 is essential for the Th2 differentiation process, and TGF- $\beta$ to TH17 and Tregs. Although $M$. capsulatus behaved more similar to E. coli than L. rhamnosus in its ability to induce cytokine production from MoDCs, both the magnitude and cytokine profiles of the two strains were different. Both strains for example induced similar levels of IL-2, but $E$. coli induced considerably higher levels of IL-23, a cytokine linked to the generation and maintenance of Th17 functions. $M$. capsulatus induced negligible IL-1 $\beta$, and compared to $E$. coli substantially less of Th1 polarizing factors IL12p70 and IFN- $\gamma$ as well as reduced IL-6 levels. IL-6 is a cytokine that plays a role in proliferation and survival of both Th1 and Th2 cells, is important for the commitment of $\mathrm{CD} 4^{+}$cells to the Th17 and Th22 lineages and has an inhibitory role in Treg development (Hunter and Jones, 2015).

Bacteria-induced differences in MoDC cytokine production were also functionally reflected in different $\mathrm{T}$ cell polarizing ability in MoDC-T cell co-cultures. In response to bacteriaprimed MoDCs, $\mathrm{T}$ cells produced increased levels of the anti-inflammatory cytokine IL-10. IL-10 plays important roles both in preventing inflammatory responses to intestinal microbiota under steady state conditions, and in limiting $\mathrm{T}$ cell-driven inflammation in pathogen clearance (Maynard and Weaver, 2008). Notably, MoDC-priming with all three bacteria significantly increased concentration of the Th1 signature cytokine IFN- $\gamma$ and reduced the levels of typical Th2 cytokines IL-13 and IL-5. Th2 development has previously been suggested to be a "default pathway" in the absence of IL-12 (Moser and Murphy, 2000). In agreement with that, in our experiments unprimed MoDCs tended to induce Th2 cell responses compared to MoDCs primed by bacteria. The observation that even the Gram-positive L. rhamnosus drives Th1 development suggest that LPS is not a critical factor in bacteria driven DC-mediated Th1 development, in support of previous reports (Smits et al., 2004).

Coherent with results from DC cytokine analysis, the cytokine profile of T cells co-incubated with MoDCs primed by Gramnegative bacteria was markedly different from that of $\mathrm{T}$ cells activated by MoDCs treated with the Gram-positive $L$. rhamnosus. Again L. rhamnosus resulted in lower levels of most of the cytokines measured, a reduction in the pro-inflammatory 

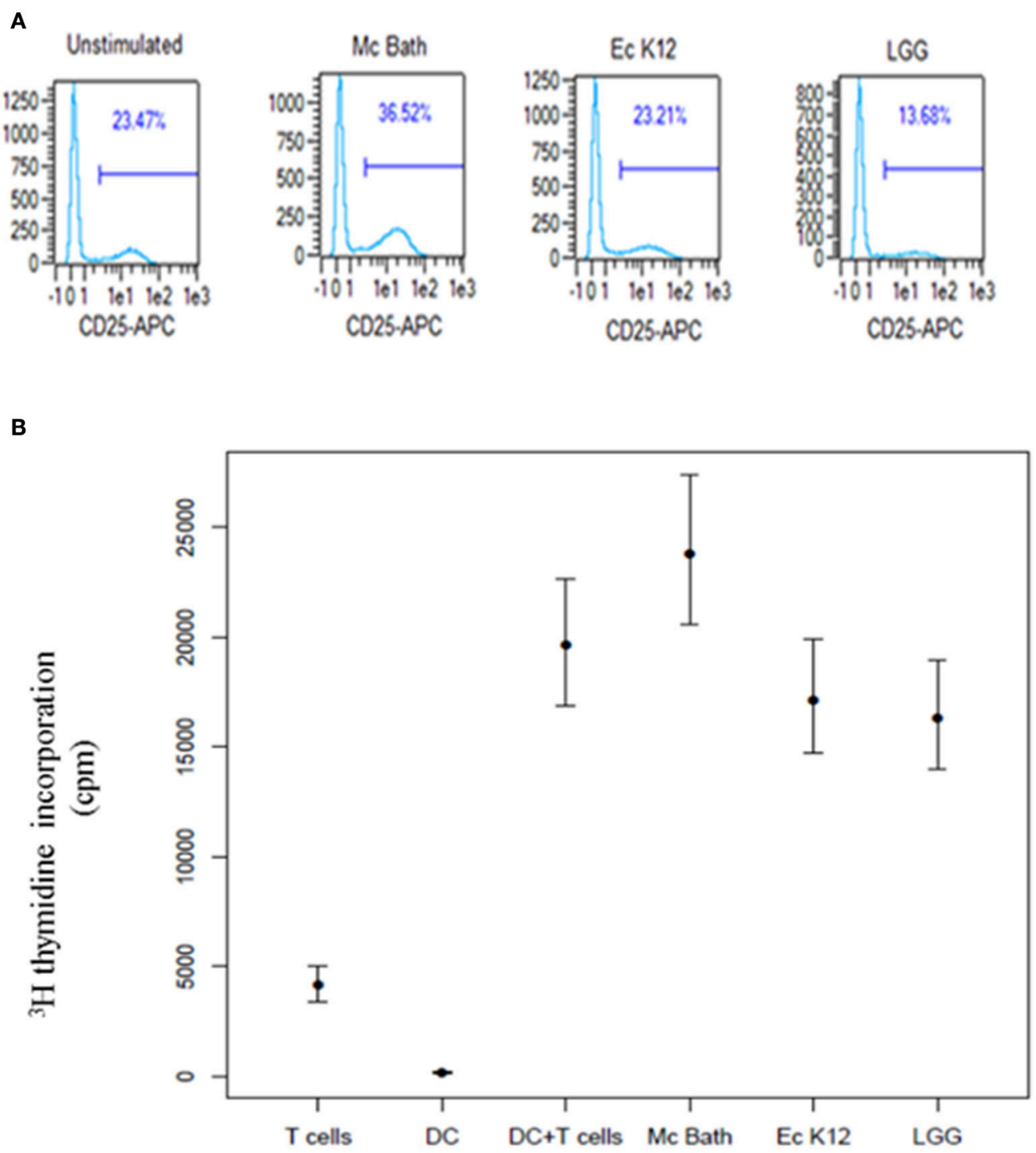

FIGURE 5 | M. capsulatus primed MoDCs efficiently induce T cell activation and proliferation. (A) Immature MoDCs were primed by UV inactivated bacteria for $24 \mathrm{~h}$ in a ratio of 1:100 (DC: bacteria). Primed MoDCs were co-incubated with allogenic T cells in the presence of IL-2. After 5 days of co-culture cells were harvested, stained for CD4 and CD25 surface protein and analyzed by flow cytometry. Cells were gated on CD4-FITC expression and the percentage of CD4+ cells expressing CD25 are shown. Plots represent results from 4 different MoDC/T cell donor combinations. (B) MoDCs primed by either UV-inactivated M. capsulatus Bath, E. coli K12, or Lactobacillus rhamnosus GG for $24 \mathrm{~h}$ were co-incubated with allogenic T cells from two different donors. After $96 \mathrm{~h}$ cells were pulsed by $1 \mu \mathrm{Ci}$ $\left[{ }^{3} \mathrm{H}\right]$ thymidine. Thymidine incorporation was determined by liquid scintillation counting $18.5 \mathrm{~h}$ after pulsing. The amount of incorporated thymidine is reported as counts per minute (cpm). Bars indicate 95\% confidence interval on values from 5 different donor combinations.

IL-18 and no increase of IL- $1 \alpha$, IL1- $\beta$, IL-6 compared to negative control. Neither did it induce cytokines typically associated with Th17/Th9/Th22 cells (IL-23, IL-17A, IL-21, IL-22, IL-9, TNF- $\alpha$ ) compared to a control of $\mathrm{T}$ cells stimulated by unprimed DC. The low T cell-levels of cytokines in response to L. rhamnosus is in agreement with a previous report showing that L. rhamnosusprimed MoDCs induce hyporesponsive T cells in DC-T cell co-cultures (Braat et al., 2004).

In contrast to L. rhamnosus $M$. capsulatus Bath, and E. coli K12 induced proinflammatory cytokines IL-6, IL- $1 \beta$, and IL- $1 \alpha$ as well as cytokines associated with generation and maintenance of the Th17 subset (IL-23, IL-17A, IL-21, IL-22), Th22 cytokines (IL-22, TNF- $\alpha$ ) and Th9 cytokines (IL-9 and IL-21). However, $M$. capsulatus induced significantly less pro-inflammatory cytokines IL1- $\alpha$, IL-1 $\beta$, and IL- 6 and anti-inflammatory IL-10. There was no significant difference in the Th1 signature cytokine IFN- $\gamma$ or Th9 cytokines IL-9 and IL-21. However, significantly less IL-23, IL-17A, and IL-22 was produced in response to $M$. capsulatus than to E. coli. The cytokine profile thus indicated that different effector cells dominate in response to the two Gramnegative bacteria. E. coli is a stronger inducer of the Th17 subset whereas $M$. capsulatus induce Th1/T9 effector cells over Th17 

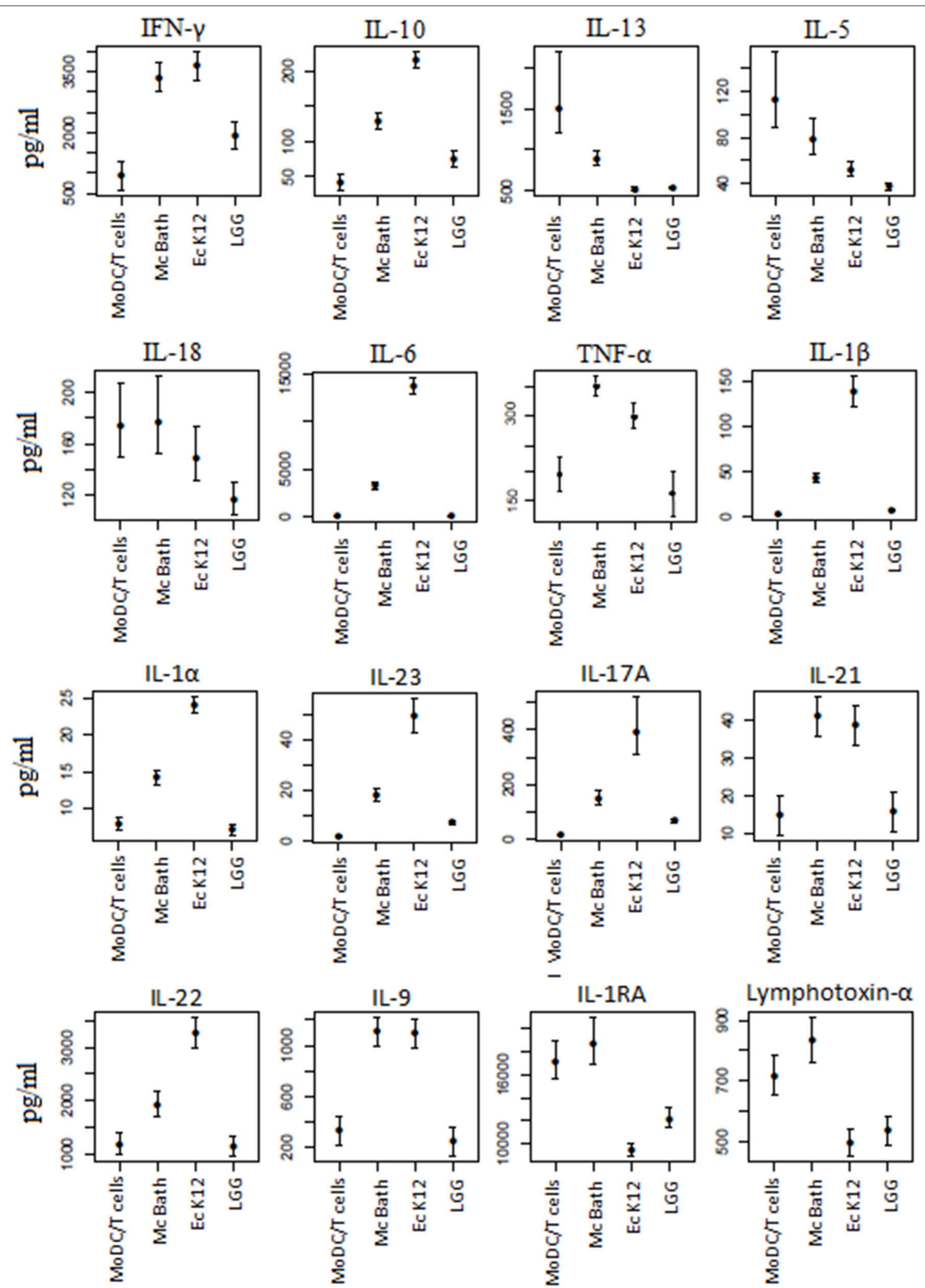

FIGURE 6 | Bacterial stimulation results in different effector T cell profiles. Unprimed MoDCs or MoDCs primed by M. capsulatus or control bacteria were co-incubated with allogenic $T$ cells for 5 days. Growth medium was collected and analyzed for cytokines by multiplex immunoassay. Bars represent $95 \%$ confidence intervals on cytokine concentrations from 4 donor combinations. 
cells in vitro. Some probiotics have been reported to induce Foxp3+ regulatory T cells (Kwon et al., 2010). It has been suggested that peripherally-induced Treg develop from naïve, $\mathrm{CD} 4+$ cells exposed to antigens under tolerogenic conditions (e.g., by immature DCs with low levels of co-stimulation) with an essential requirement for TGF- $\beta$ signaling (Marie et al., 2005; Johnston et al., 2016). We did not find detectable levels of TGF$\beta$ released from MoDC stimulated by $M$. capsulatus. Neither did we find significantly increased expression of Foxp3 in T cell co-cultures with bacteria stimulated MoDC (data not shown).

E. coli and L. rhamnosus, but not M. capsulatus Bath, reduced lymphotoxin- $\alpha$ and IL-1RA in culture supernatants. Lymphotoxin- $\alpha$ is important for lymphoid organ development, regulates $\mathrm{T}$ cell homing and IgA production in the gut and contributes to shaping the gut microbiome (Ruddle, 2014). The balance between IL-1 and IL-1RA in local tissues plays an important role in the susceptibility and severity of a number of diseases, including IBD (Arend, 2002). For example, significant decrease in the intestinal mucosal IL-1RA/IL-1 ratio has been found in freshly isolated intestinal mucosal cells, and in mucosal biopsies obtained from both Crohn's disease and ulcerative colitis patients as compared to control subjects (Casini-Raggi et al., 1995). The observation that IL- $1 \alpha$ and IL- $1 \beta$ is reduced, while IL1RA is kept high in M. capsulatus primed DC-T cell co-cultures is interesting in the light of $M$. capsulatus anti-inflammatory effects in a murine enteritis model (Kleiveland et al., 2013). Screening for cytokine profiles associated with specific $\mathrm{T}$ effector cell populations may be a useful first step to identify strains with potential pro- or anti-inflammatory properties e.g., for further mechanistic investigation (Papadimitriou et al., 2015). It is important however to notice the limitations of in vitro systems on making in vivo predictions. Although the bacteria tested here induced different effects in $\mathrm{T}$ cells in vitro, caution should be exercised in drawing conclusions both about the direction of $\mathrm{T}$ cell polarization by these bacteria and the functional relevance in vivo. $\mathrm{T}$ cell differentiation occurs in a finely tuned manner dependent on a variety of tissue factors and cytokines, and in vitro systems cannot necessarily reflect the complex cytokine environment of the gut. For example, TGF- $\beta$ a cytokine abundant in the intestines, was not detected in our MoDC supernatants. TGF- $\beta$ is not only involved in development of

\section{REFERENCES}

Agency USEP (1997). Escherichia coli K-12 Derivatives Final Risk Assessment Attachment I. Biotechnology Program under the Toxic Substances Control Act (TSCA).

Arend, W. P. (2002). The balance between IL-1 and IL-1Ra in disease. Cytokine Growth Factor Rev. 13, 323-340. doi: 10.1016/S1359-6101(02)00020-5

Bermudez-Brito, M., Plaza-Diaz, J., Munoz-Quezada, S., Gomez-Llorente, C., and Gil, A. (2012). Probiotic mechanisms of action. Ann. Nutr. Metab. 61, 160-174. doi: $10.1159 / 000342079$

Bienenstock, J., Gibson, G., Klaenhammer, T. R., Walker, W. A., and Neish, A. S. (2013). New insights into probiotic mechanisms: a harvest from functional and metagenomic studies. Gut Microbes 4, 94-100. doi: 10.4161/gmic. 23283
Tregs, Th9 and Th17 effector cells, but it also suppresses Th1 and Th2 cell differentiation (Zheng, 2013). TGF- $\beta$ is produced by $\mathrm{CD} 103^{+}$DC (Coombes et al., 2007) a DC subset common in the intestines and is expected to play a prominent role in regulating mucosal immunity (Ruane and Lavelle, 2011). The results of bacterial priming in vitro may thus be expected to have different outcomes in an in vivo situation. The impact of immune modulatory effects of $M$. capsulatus on DC in maintaining intestinal homeostasis thus remains to be determined (study in preparation).

\section{CONCLUDING REMARKS}

Environmental bacteria, although immensely numerous and diverse, have remained largely unexplored for their immunomodulatory properties. Our results demonstrate the direct binding and functional effects of a soil bacterium on human monocyte-derived dendritic cells. The same bacterium has recently been shown to possess anti-inflammatory properties in a murine colitis model. The identification of anti-inflammatory and immunomodulatory properties of this bacterium was serendipitous. In fact, such properties may not be a rare trait of this particular soil bacterium, but rather a common feature of many environmental bacteria. Our study thus emphasizes the need to scrutinize, identify, and understand possible physiological consequences of environmental microbehost interactions, and we suggests that bacteria from soil and water should receive increased attention for their potential health benefits.

\section{AUTHOR CONTRIBUTIONS}

SI contributed to design of the work, acquisition, analysis, and interpretation of data and drafted the work. TL and CK contributed to design of the work, interpretation of data and revising work critically for important intellectual content. RH contributed to data analysis and revising work critically for important intellectual content. MJ contributed to interpretation of data and revising work critically for important intellectual content. All authors approved final version and agreed to be accountable for all aspects of the work.
Blattner, F. R., Plunkett, G. III., Bloch, C. A., Perna, N. T., Burland, V., Riley, M., et al. (1997). The complete genome sequence of Escherichia coli K-12. Science 277, 1453-1462. doi: 10.1126/science.277.5331.1453

Braat, H., van den Brande, J., van Tol, E., Hommes, D., Peppelenbosch, M., and van Deventer, S. (2004). Lactobacillus rhamnosus induces peripheral hyporesponsiveness in stimulated $\mathrm{CD} 4+\mathrm{T}$ cells via modulation of dendritic cell function. Am. J. Clin. Nutr. 80, 1618-1625. Available online at: http://ajcn. nutrition.org/content/80/6/1618.long

Brown, E. M., Sadarangani, M., and Finlay, B. B. (2013). The role of the immune system in governing host-microbe interactions in the intestine. Nat. Immunol. 14, 660-667. doi: 10.1038/ni.2611

Casadevall, A., and Pirofski, L. A. (2007). Accidental virulence, cryptic pathogenesis, martians, lost hosts, and the pathogenicity of environmental microbes. Eukaryotic Cell 6, 2169-2174. doi: 10.1128/EC.00308-07 
Casini-Raggi, V., Kam, L., Chong, Y. J., Fiocchi, C., Pizarro, T. T., and Cominelli, F. (1995). Mucosal imbalance of IL-1 and IL-1 receptor antagonist in inflammatory bowel disease. A novel mechanism of chronic intestinal inflammation. J. Immunol. 154, 2434-2440.

Coombes, J. L., Siddiqui, K. R., Arancibia-Carcamo, C. V., Hall, J., Sun, C. M., Belkaid, Y., et al. (2007). A functionally specialized population of mucosal $\mathrm{CD}_{103}{ }^{+}$DCs induces Foxp3 ${ }^{+}$regulatory $\mathrm{T}$ cells via a TGF$\beta$ and retinoic acid-dependent mechanism. J. Exp. Med. 204, 1757-1764. doi: 10.1084/jem.20070590

Dalod, M., Chelbi, R., Malissen, B., and Lawrence, T. (2014). Dendritic cell maturation: functional specialization through signaling specificity and transcriptional programming. EMBO J. 33, 1104-1116. doi: 10.1002/embj.201488027

Foligne, B., Zoumpopoulou, G., Dewulf, J., Ben Younes, A., Chareyre, F., Sirard, J. C., et al. (2007). A key role of dendritic cells in probiotic functionality. PLoS ONE 2:e313. doi: 10.1371/journal.pone.0000313

Hunter, C. A., and Jones, S. A. (2015). IL-6 as a keystone cytokine in health and disease. Nat. Immunol. 16, 448-457. doi: 10.1038/ni.3153

Indrelid, S., Mathiesen, G., Jacobsen, M., Lea, T., and Kleiveland, C. R. (2014). Computational and experimental analysis of the secretome of Methylococcus capsulatus (Bath). PLoS ONE 9:e114476. doi: 10.1371/journal.pone.01 14476

Johnston, C. J., Smyth, D. J., Dresser, D. W., and Maizels, R. M. (2016). TGF- $\beta$ in tolerance, development and regulation of immunity. Cell. Immunol. 299, 14-22. doi: 10.1016/j.cellimm.2015.10.006

Kapsenberg, M. L. (2003). Dendritic-cell control of pathogen-driven T-cell polarization. Nat. Rev. Immunol. 3, 984-993. doi: 10.1038/nri1246

Kleiveland, C. R., Hult, L. T., Spetalen, S., Kaldhusdal, M., Christofferesen, T. E., Bengtsson, O., et al. (2013). The noncommensal bacterium Methylococcus capsulatus (Bath) ameliorates dextran sulfate (Sodium Salt)-Induced Ulcerative Colitis by influencing mechanisms essential for maintenance of the colonic barrier function. Appl. Environ. Microbiol. 79, 48-56. doi: 10.1128/AEM.02464-12

Kwon, H. K., Lee, C. G., So, J. S., Chae, C. S., Hwang, J. S., Sahoo, A., et al. (2010). Generation of regulatory dendritic cells and $\mathrm{CD} 4^{+}$Foxp $^{+} \mathrm{T}$ cells by probiotics administration suppresses immune disorders. Proc. Natl. Acad. Sci. U.S.A. 107, 2159-2164. doi: 10.1073/pnas.0904055107

Lebeer, S., Vanderleyden, J., and De Keersmaecker, S. C. (2010). Host interactions of probiotic bacterial surface molecules: comparison with commensals and pathogens. Nat. Rev. Microbiol. 8, 171-184. doi: 10.1038/nrmicro 2297

Mann, E. R., Landy, J. D., Bernardo, D., Peake, S. T., Hart, A. L., AlHassi, H. O., et al. (2013). Intestinal dendritic cells: their role in intestinal inflammation, manipulation by the gut microbiota and differences between mice and men. Immunol. Lett. 150, 30-40. doi: 10.1016/j.imlet.2013. 01.007

Marie, J. C., Letterio, J. J., Gavin, M., and Rudensky, A. Y. (2005). TGF$\beta 1$ maintains suppressor function and Foxp3 expression in $\mathrm{CD} 4{ }^{+} \mathrm{CD} 25^{+}$ regulatory T cells. J. Exp. Med. 201, 1061-1067. doi: 10.1084/jem.20042276

Maynard, C. L., and Weaver, C. T. (2008). Diversity in the contribution of interleukin-10 to T-cell-mediated immune regulation. Immunol. Rev. 226, 219-233. doi: 10.1111/j.1600-065X.2008.00711.x

Mileti, E., Matteoli, G., Iliev, I. D., and Rescigno, M. (2009). Comparison of the immunomodulatory properties of three probiotic strains of Lactobacilli using complex culture systems: prediction for in vivo efficacy. PLoS ONE 4:e7056. doi: 10.1371/journal.pone.0007056

Moser, M., and Murphy, K. M. (2000). Dendritic cell regulation of TH1-TH2 development. Nat. Immunol. 1, 199-205. doi: 10.1038/79734

Nicholson, J. K., Holmes, E., Kinross, J., Burcelin, R., Gibson, G., Jia, W., et al. (2012). Host-gut microbiota metabolic interactions. Science 336, 1262-1267. doi: $10.1126 /$ science. 1223813

Papadimitriou, K., Zoumpopoulou, G., Foligne, B., Alexandraki, V., Kazou, M., Pot, B., et al. (2015). Discovering probiotic microorganisms: in vitro, in vivo, genetic and omics approaches. Front. Microbiol. 6:58. doi: $10.3389 /$ fmicb. 2015.00058

Pils, M. C., Bleich, A., Prinz, I., Fasnacht, N., Bollati-Fogolin, M., Schippers, A., et al. (2011). Commensal gut flora reduces susceptibility to experimentally induced colitis via T-cell-derived interleukin-10. Inflamm. Bowel Dis. 17, 2038-2046. doi: 10.1002/ibd.21587

Qiu, X., Zhang, M., Yang, X., Hong, N., and Yu, C. (2013). Faecalibacterium prausnitzii upregulates regulatory $\mathrm{T}$ cells and anti-inflammatory cytokines in treating TNBS-induced colitis. J. Crohns. Colitis 7, e558-e568. doi: 10.1016/j.crohns.2013.04.002

Reis e Sousa, C. (2006). Dendritic cells in a mature age. Nat. Rev. Immunol. 6, 476-483. doi: 10.1038/nri1845

Rook, G. A. (2007). The hygiene hypothesis and the increasing prevalence of chronic inflammatory disorders. Trans. R. Soc. Trop. Med. Hyg. 101, 1072-1074. doi: 10.1016/j.trstmh.2007.05.014

Rook, G. A. (2010). 99th Dahlem conference on infection, inflammation and chronic inflammatory disorders: darwinian medicine and the 'hygiene' or 'old friends' hypothesis. Clin. Exp. Immunol. 160, 70-79. doi: 10.1111/j.1365-2249.2010.04133.x

Rook, G. A., Adams, V., Hunt, J., Palmer, R., Martinelli, R., and Brunet, L. R. (2004). Mycobacteria and other environmental organisms as immunomodulators for immunoregulatory disorders. Springer Semin. Immunopathol. 25, 237-255. doi: 10.1007/s00281-003-0148-9

Ruane, D. T., and Lavelle, E. C. (2011). The role of $\mathrm{CD}_{103}{ }^{+}$dendritic cells in the intestinal mucosal immune system. Front. Immunol. 2:25. doi: 10.3389/fimmu.2011.00025

Ruddle, N. H. (2014). Lymphotoxin and TNF: how it all began-a tribute to the travelers. Cytokine Growth Factor Rev. 25, 83-89. doi: 10.1016/j.cytogfr.2014.02.001

Sang, L. X., Chang, B., Dai, C., Gao, N., Liu, W. X., and Jiang, M. (2014). Heat-killed VSL\#3 ameliorates dextran sulfate sodium (DSS)-induced acute experimental colitis in rats. Int. J. Mol. Sci. 15, 15-28. doi: 10.3390/ijms150 10015

Schreibelt, G., Tel, J., Sliepen, K. H., Benitez-Ribas, D., Figdor, C. G., Adema, G. J., et al. (2010). Toll-like receptor expression and function in human dendritic cell subsets: implications for dendritic cell-based anti-cancer immunotherapy. Cancer Immunol. Immunother. 59, 1573-1582. doi: 10.1007/s00262-0100833-1

Schuijs, M. J., Willart, M. A., Vergote, K., Gras, D., Deswarte, K., Ege, M. J., et al. (2015). Farm dust and endotoxin protect against allergy through A20 induction in lung epithelial cells. Science 349, 1106-1110. doi: 10.1126/science.aac 6623

Smith, K. A. (1988). Interleukin-2: inception, impact, and implications. Science 240, 1169-1176. doi: 10.1126/science.3131876

Smits, H. H., van Beelen, A. J., Hessle, C., Westland, R., de Jong, E., Soeteman, E., et al. (2004). Commensal Gram-negative bacteria prime human dendritic cells for enhanced IL-23 and IL-27 expression and enhanced Th1 development. Eur. J. Immunol. 34, 1371-1380. doi: 10.1002/eji.200324815

Sommer, F., and Backhed, F. (2013). The gut microbiota-masters of host development and physiology. Nat. Rev. Microbiol. 11, 227-238. doi: 10.1038/nrmicro2974

Souza, E. L., Elian, S. D., Paula, L. M., Garcia, C. C., Vieira, A. T., Teixeira, M. M., et al. (2016). Escherichia coli strain Nissle 1917 ameliorates experimental colitis by modulating intestinal permeability, the inflammatory response and clinical signs in a faecal transplantation model. J. Med. Microbiol. 65, 201-210. doi: $10.1099 /$ jmm.0.000222

Steinman, R. M. (2012). Decisions about dendritic cells: past, present, and future. Annu. Rev. Immunol. 30, 1-22. doi: 10.1146/annurev-immunol-100311-102839

Strachan, D. P. (1989). Hay fever, hygiene, and household size. BMJ 299, 1259-1260. doi: 10.1136/bmj.299.6710.1259

Strachan, D. P. (2000). Family size, infection and atopy: the first decade of the "hygiene hypothesis." Thorax 55(Suppl. 1), S2-S10. doi: 10.1136/thorax.55.suppl_1.S2

Toumi, R., Soufli, I., Rafa, H., Belkhelfa, M., Biad, A., and TouilBoukoffa, C. (2014). Probiotic bacteria lactobacillus and bifidobacterium attenuate inflammation in dextran sulfate sodium-induced experimental colitis in mice. Int. J. Immunopathol. Pharmacol. 27, 615-627. doi: 10.1177/039463201402700418

Turnbaugh, P. J., Ley, R. E., Hamady, M., Fraser-Liggett, C. M., Knight, R., and Gordon, J. I. (2007). The human microbiome project. Nature 449, 804-810. doi: $10.1038 /$ nature 06244 
Verhasselt, V., Buelens, C., Willems, F., De Groote, D., Haeffner-Cavaillon, N., and Goldman, M. (1997). Bacterial lipopolysaccharide stimulates the production of cytokines and the expression of costimulatory molecules by human peripheral blood dendritic cells: evidence for a soluble CD14-dependent pathway. J. Immunol. 158, 2919-2925.

Whittenbury, R., Phillips, K. C., and Wilkinson, J. F. (1970). Enrichment, isolation and some properties of methane-utilizing bacteria. J. Gen. Microbiol. 61, 205-218. doi: 10.1099/00221287-61-2-205

Zheng, S. G. (2013). Regulatory T cells vs Th17: differentiation of Th17 versus Treg, are the mutually exclusive? Am. J. Clin. Exp. Immunol. 2, 94-106. doi: 10.1007/ 978-3-0348-0522-3_6
Conflict of Interest Statement: The authors declare that the research was conducted in the absence of any commercial or financial relationships that could be construed as a potential conflict of interest.

Copyright $\odot 2017$ Indrelid, Kleiveland, Holst, Jacobsen and Lea. This is an openaccess article distributed under the terms of the Creative Commons Attribution License (CC BY). The use, distribution or reproduction in other forums is permitted, provided the original author(s) or licensor are credited and that the original publication in this journal is cited, in accordance with accepted academic practice. No use, distribution or reproduction is permitted which does not comply with these terms. 